\title{
Free Vibration Analysis of Rotating Plates in High Thermal Environments Using the Finite Element Method
}

\author{
Inala Ramu and Sukesh C. Mohanty \\ Mechanical Engineering Department, NIT, Rourkela-769008, India
}

(Received 11 November 2014; accepted: 30 July 2015)

\begin{abstract}
The aim of the work is to study the vibration characteristics of rotating functionally graded material (FGM) plates using the finite element method. The mechanical properties of the ordinary and temperature dependent FGM plates are assumed to be varying in the thickness direction according to a power law distribution in terms of the volume fractions of the constituents. The temperature field is ascertained using a nonlinear distribution in the thickness direction only. The present method results are compared with numerical results available in the literature. The frequency characteristics of the rotating ordinary FGM and temperature dependent FGM plates are examined. The effect of parameters such as hub radius, rotating speed, temperature change, and power law index value on the natural frequencies of rotating FGM plates are investigated through this study. The plots reveal that the temperature field, rotational speed, and the gradient index in the material properties have a significant effect on the vibration characteristics of the rotating ordinary FGM and temperature dependent FGM plates.
\end{abstract}

\section{INTRODUCTION}

FGMs are advanced composite materials made of two or more constituent's phases with continuous and smoothly varying composition. Many rotating structures such as turbine blades, wind turbine blades, and helicopter rotor blades can be modelled as rotating plates. Due to the extensive use of rotating plates, FGM plates are of significant interest to many researchers in the field of modelling, analysis, and design. Acquaintance with the natural frequencies of these rotating plate structures is essential in the design stages for studying their dynamical behaviour under resonance. The frequency analysis of rotating plates often varies considerably from those of non-rotating plates due to the influence of centrifugal inertia force. Due to this, there is a considerable variation of vibration characteristics that result from rotation.

The free vibration characteristics of rotating cantilever isotropic plates have been studied in several investigations. The Coriolis effect on the first bending and first torsional frequencies of flat rotating low aspect ratio cantilever plates using the finite element method has been investigated by Ramamurti and Kielb. ${ }^{1}$ Murthy and Ramamurti presented the eigen frequencies of twisted rotating plates by using two different shape functions. ${ }^{2}$ Here, the method of stress smoothing has been used for reducing numerical errors when calculating the improved stiffness matrix. The free vibration analysis of rotating rectangular plates has been studied by Wang et al. ${ }^{3}$ Yoo et al. derived the equation of motion for the vibration analysis of a rotating pre-twisted blades. ${ }^{4}$ They have used a modelling method that employs hybrid deformation variables. The linear equations of motion for the flap wise bending vibration analysis of rotating plates was developed by Yoo and Kim. ${ }^{5}$ They used two in-plane stretch variables by which the exact in-plane strain energy of a plate can be expressed in a quadratic form.
Yoo and Pierre have investigated the model characteristics of a rotating cantilever plate by dynamic modelling method. ${ }^{6}$ In this method, the parameters have been identified through a dimensional analysis and the effects of the parameters on the variations of model characteristics have also been studied. A modelling method for the modal analysis of cantilever plates undergoing accelerated in-plane motion has been presented by Lim and Yoo. ${ }^{7}$ In their analysis, Von Karman strain measures were used to derive the in-plane and the lateral equations of motion. Finite element formulation for vibration analysis of rotating thick plates has been reported by Hashemi et al. ${ }^{8}$ They used the Kane dynamic method for the derivation of nonlinear governing equations of motion, which included Coriolis effects and the couplings between in-plane and out of plane deformations.

A theoretical analysis of the FGM thin plates based on the physical neutral surface has been studied by Zhang and Zhou. ${ }^{9}$ They assumed changeable parameters such as Young's modulus and the mass density along the thickness of the plate, the physical neutral surface of an FGM plate is determined by the theory of thin plate, when Poisson's ratio is assumed to be constant in the whole plate. The effects of loading conditions and the aspect ratio on the mechanical behaviour of an FGM plate have been discussed by Chi and Chung. ${ }^{10}$ They gave a comparison of the results of Polynomial-FGM, Sigmoidal-FGM, and Exponential-FGM. Croce and Venini formulated the governing equations for rectangular plates made of FGMs using the variational approach. ${ }^{11}$ The free vibration and static analysis of FGM plates using higher order shear deformation theory and with a special modification in the transverse displacement in conjunction with finite element models have been proposed by Talha and Singh. ${ }^{12}$ A new exact closed form approach was presented by Hashemi et al. for free vibration analysis of thick rectangular FG plates using the third-order shear deformation 


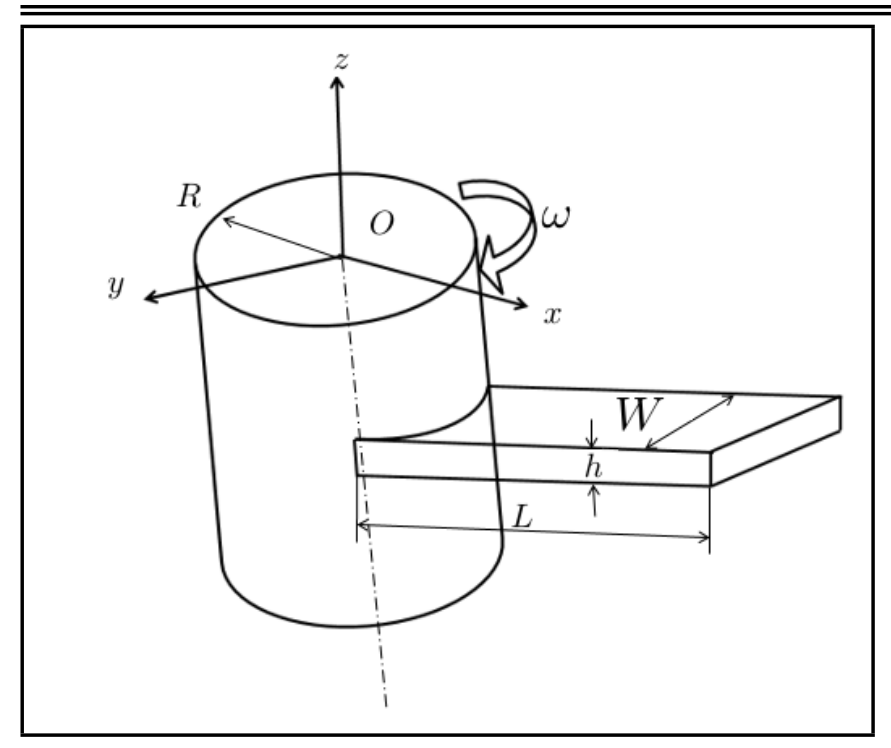

Figure 1. A rotating cantilever FGM plate.

theory of Reddy. ${ }^{13}$

An analytical solution for nonlinear free and forced vibration of FGM plates in thermal environment has been developed by Huang and Shen. ${ }^{14}$ Here the temperature field was assumed to be constant in the plane and only varies in the thickness direction of the plate. Yang and Shen ${ }^{15}$ have proposed a theoretical method for vibration characteristics study of initially stressed functionally graded rectangular plates made up of metal and ceramic in the thermal environment. The thermomechanical induced vibration characteristics of FGM plates have been studied by Talha and Singh. ${ }^{16}$ In this work, their objective was to develop a higher order shear deformation theory with an appropriate modification in transverse displacement that provides an additional freedom to the displacements through the thickness and thus eliminates the over prediction. Vibration analysis of temperature dependent functionally graded rectangular plates in thermal environment has been explained by Young-Wann Kim. ${ }^{17}$ Dynamic stability of FGM plates in high temperature environment has been studied by Ramu and Mohanty. ${ }^{18}$ Exact solution for free vibration analysis of variable thickness thick annular FGM plates was studied by Efraim and Eisenberger. ${ }^{19}$

According to our assessment of the published literature, free vibration analysis of a rotating FGM plate in high thermal environment needs of further exploration. The proposed work studies the effects of parameters such as the angular speed, hub ratio, index values, and temperature change on the free vibration characteristics of the rotating FGM plate in high thermal surroundings.

\section{MATHEMATICAL FORMULATION}

\subsection{Theoretical Background}

Consider a cantilever FGM plate of a length $L$, width $W$, and thickness $h$, which is rigidly attached to the hub with a radius $R$, as shown in Fig. 1. The plate is modelled assuming the first order shear deformation theory and it has material properties varying along the thickness direction. Thus, the transverse shear effects are considered in the formulation.

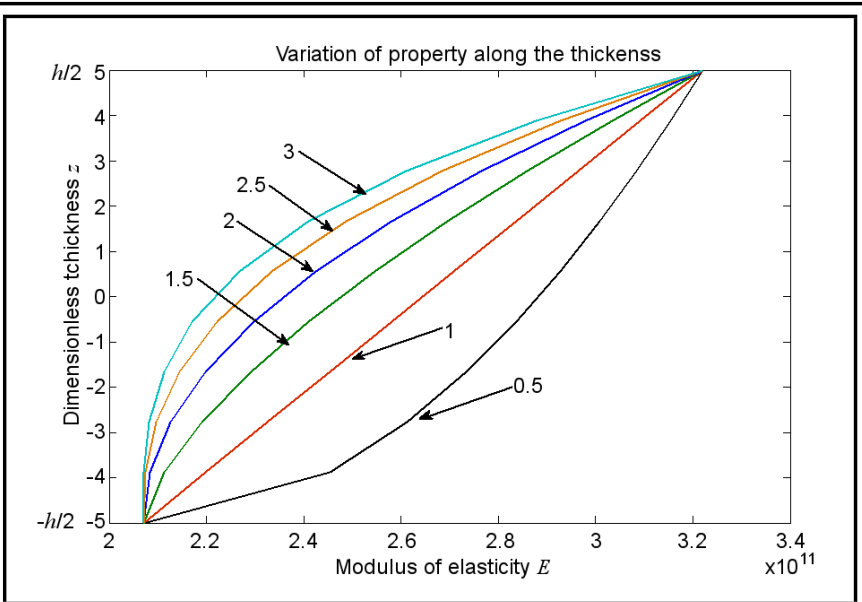

Figure 2. Variation of the modulus of elasticity through the thickness.

\subsection{Functionally Graded Material Plate Constitutive Law}

An FGM plate is made of metal and ceramic mixtures, the material composition varies from the bottom to the top surface. The top surface $(z=h / 2)$ of the plate is ceramic-rich, whereas the bottom surface ( $z=h / 2)$ is metal-rich. The effective material properties are assumed to vary through the thickness direction of the plate according to the simple power law. The effective material properties can be expressed as:

$$
P=P_{c} V_{c}+P_{m} V_{m}
$$

where $P_{c}$ and $P_{m}$ are the material properties of the ceramic and metal phase respectively. $V_{c}$ and $V_{m}$ are the volume fraction and metal phase and they are related as:

$$
V_{C}(Z)+V_{M}(Z)=1
$$

The volume fraction of the constituent ceramic material as per a power law can be expressed as

$$
V_{c}=\left(\frac{2 \times z+h}{2 \times h}\right)^{k}, \quad 0 \leq k \leq \infty ;
$$

where $k$ is the power law index, which prescribes the constituent material variation along the thickness direction of the plate. The effective material properties such as Youngs modulus, Poisson's ratio, and mass density of an ordinary FGM plate can be expressed based on the volume fraction of the constituent materials, according to a simple power law function as:

$$
\begin{aligned}
& E(z)=E_{m}+\left(E_{c}-E_{m}\right)\left(\frac{1}{2}+\frac{z}{h}\right)^{k} \\
& \rho(z)=\rho_{m}+\left(\rho_{c}-\rho_{m}\right)\left(\frac{1}{2}+\frac{z}{h}\right)^{k} \\
& v(z)=v_{m}+\left(v_{c}-v_{m}\right)\left(\frac{1}{2}+\frac{z}{h}\right)^{k}
\end{aligned}
$$

For an FGM plate made up of steel and alumina, Fig. 2 shows the variation of the modulus of elasticity through the dimensionless thickness.

\subsection{Temperature Dependent FGM Properties}

The elastic modulus $E$, the mass density $\rho$, Poisson's ratio $\nu$, thermal conductivity $K$, and the coefficient of thermal expansion $\alpha$ are temperature-dependent material properties. The 


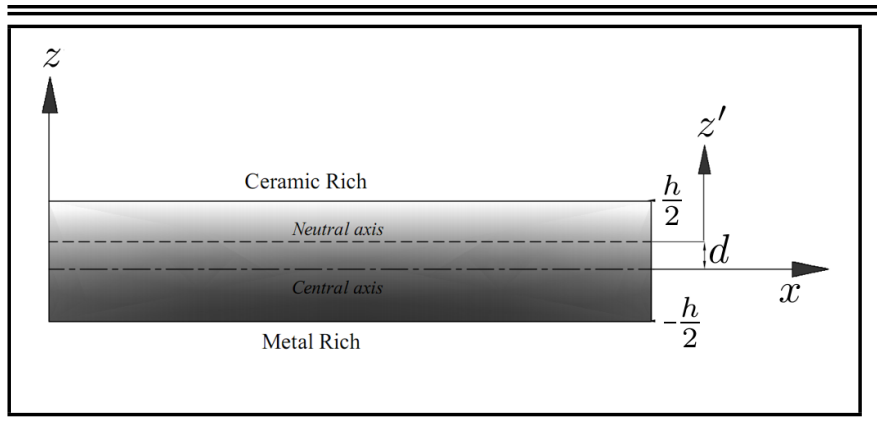

Figure 3. Geometry of the FGM plate.

temperature-dependent material properties are obtained using the following expression by Praveen and Reddy. ${ }^{20}$

$$
R(T)=P_{0}\left(P_{-1} T^{-1}+1+P_{1} T+P_{2} T^{2}+P_{3} T^{3}\right)
$$

where $P_{0}, P_{1}, P_{2}$, and $P_{3}$ are the coefficients of temperature $T$ in Kelvin and are unique to each constituent. $T=T_{0}+T(z)$, where $T(z)$ is temperature rise through the thickness direction and $T_{0}$ is room temperature. From the above equations, the effective material properties with two constituents for FGM plates can be expressed as follows:

$$
\begin{array}{r}
E(z, T)=E_{m}(T)+\left[E_{c}(T)-E_{m}(T)\right]\left(\frac{2 z+h}{2 h}\right)^{k} ; \\
\alpha(z, T)=\alpha_{m}(T)+\left[\alpha_{c}(T)-\alpha_{m}(T)\right]\left(\frac{2 z+h}{2 h}\right)^{k} ; \\
\nu(z, T)=\nu_{m}(T)+\left[\nu_{c}(T)-\nu_{m}(T)\right]\left(\frac{2 z+h}{2 h}\right)^{k} ; \\
\rho(z)=\rho_{m}+\left[\rho_{c}-\rho_{m}\right]\left(\frac{2 z+h}{2 h}\right)^{k} ; \\
K(z)=K_{m}+\left[K_{c}-K_{m}\right]\left(\frac{2 z+h}{2 h}\right)^{k} .
\end{array}
$$

During the analysis, the temperature field was applied in the thickness direction only and the temperature field was assumed to be constant in the XYplane of the plate.

\subsection{Physical Neutral Surface of the FGM Plate}

For plate made of FGM (Fig. 3), the neutral surface does not coincide with its geometric mid-surface. The distance $(d)$ of the neutral surface from the geometric mid-surface may be expressed as:

$$
d=\frac{\int_{-h / 2}^{h / 2} z E(z, T) d z}{\int_{-h / 2}^{h / 2} E(z, T) d z} .
$$

\subsection{Nonlinear Temperature Distribution}

A steady-state heat transfer equation used to obtain the temperature distribution along the thickness, can be represented as:

$$
-\frac{d}{d z}\left[K\left(z^{\prime}\right) \frac{d T}{d z}\right]=0
$$

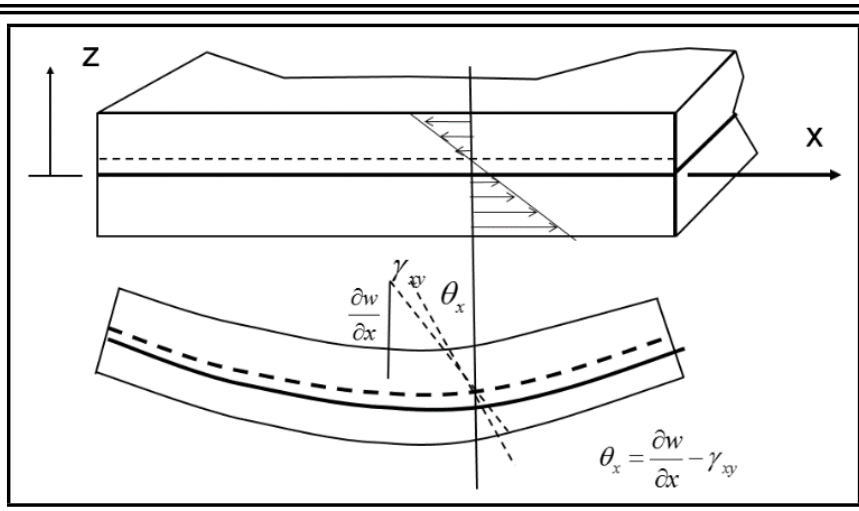

Figure 4. Plate structure before and after deformation.

This equation is solved by prescribing temperature load at top and bottom surfaces such as $T=T_{c}$ at $z^{\prime}=h / 2-d$ and $T=T_{m}$ at $z^{\prime}=h / 2-d$. The temperature distribution along the thickness direction can be obtained as:

$$
T\left(z^{\prime}\right)=T_{m}+\left(T_{c}-T_{m}\right) \frac{\int_{-h / 2-d}^{z^{\prime}} \frac{1}{h / 2-d} d z^{\prime}}{\int_{-h / 2-d} \frac{1}{k\left(z^{\prime}\right)} d z^{\prime}} .
$$

\subsection{Kinematics}

Plate structures made of graded materials are characterized by non-negligible shear deformation so that the extension of the classical model suggested by Croce and Venini ${ }^{11}$ to the case of graded material plates provides a good compromise between numerical accuracy and computational load. The displacements $u, v, w$ at a point $\left(x, y, z^{\prime}\right)$ from the neutral plane of an FGM plate (Fig. 4) can be expressed as:

$$
\begin{aligned}
& u(x, y, z)=z^{\prime} \theta_{x}(x, y), \\
& v(x, y, z)=z^{\prime} \theta_{y}(x, y), \\
& w(x, y, z)=w(x, y)
\end{aligned}
$$

where $\theta_{x}$ and $\theta_{y}$ the rotations of the normal in xz and yz planes, respectively.

In-plane strain and transverse shear strains $\gamma_{x}$ and $\gamma_{y}$ are conversely constant through the thickness and are defined as:

$$
\left[\begin{array}{c}
\varepsilon_{x x} \\
\varepsilon_{y y} \\
\varepsilon_{x y}
\end{array}\right]=z^{\prime}\left[\begin{array}{c}
\theta_{x, x} \\
\theta_{y, y} \\
\theta_{x, y}+\theta_{y, x}
\end{array}\right],\left[\begin{array}{l}
\gamma_{x} \\
\gamma_{y}
\end{array}\right]=\left[\begin{array}{c}
w_{, x}+\theta_{x} \\
w_{, y}+\theta_{y}
\end{array}\right]
$$

The plane stress and transverse shear stress relationship are given as:

$$
\left[\begin{array}{c}
\sigma_{x} \\
\sigma_{y} \\
\sigma_{x y}
\end{array}\right]=z^{\prime} D_{b}\left[\begin{array}{l}
\varepsilon_{x x} \\
\varepsilon_{y y} \\
\varepsilon_{x y}
\end{array}\right],\left[\begin{array}{c}
\tau_{x} \\
\tau_{y}
\end{array}\right]=D_{s}\left[\begin{array}{l}
\gamma_{x} \\
\gamma_{y}
\end{array}\right]
$$

where $D_{b}=\frac{E h^{3}}{12\left(1-\nu^{2}\right)}\left[\begin{array}{ccc}1 & \nu & 0 \\ \nu & 1 & 0 \\ 0 & 0 & \frac{1-\nu}{2}\end{array}\right], \quad D_{s}=$ $\frac{E h k_{s h}}{2(1-\nu)}\left[\begin{array}{ll}1 & 0 \\ 0 & 1\end{array}\right]$ 
Bending moment and shear stress relationships are:

$$
\begin{gathered}
{\left[\begin{array}{l}
M_{x} \\
M_{y} \\
M_{x y}
\end{array}\right]=\int_{-h / 2-d}^{h / 2-d}\left[\begin{array}{c}
\sigma_{x} \\
\sigma_{y} \\
\sigma_{x y}
\end{array}\right] z^{\prime} d z^{\prime}=} \\
{\left[\begin{array}{c}
\theta_{x, x} \\
\theta_{y, y} \\
\theta_{x, y}+\theta_{y, x}
\end{array}\right] \int_{-h / 2-d}^{h / 2-d} D z^{\prime 2} d z^{\prime} .} \\
{\left[\begin{array}{c}
Q_{x} \\
Q_{y}
\end{array}\right]=\int_{-h / 2-d}^{h / 2-d}\left[\begin{array}{c}
\tau_{x} \\
\tau_{y}
\end{array}\right] d z^{\prime}} \\
=\left[\begin{array}{l}
w_{, x}+\theta_{x} \\
w_{, y}+\theta_{y}
\end{array}\right] \int_{-h / 2-d}^{h / 2-d} D_{s} d z^{\prime} .
\end{gathered}
$$

\subsubsection{Constitutive Relations}

The linear thermo-mechanical constitutive relations are:

$$
\begin{aligned}
& \left\{\begin{array}{c}
\sigma_{x x} \\
\sigma_{\mathrm{yy}} \\
\tau_{x y}
\end{array}\right\}= \\
& {\left[\begin{array}{ccc}
Q_{11} & Q_{12} & 0 \\
Q_{21} & Q_{22} & 0 \\
0 & 0 & Q_{66}
\end{array}\right]\left(\left\{\begin{array}{c}
\varepsilon_{\mathrm{x}} \\
\varepsilon_{\mathrm{y}} \\
\gamma_{x y}
\end{array}\right\}-\left\{\begin{array}{l}
1 \\
1 \\
0
\end{array}\right\} \alpha\left(z^{\prime}, T\right)\right)}
\end{aligned}
$$

where $Q_{11}=\frac{E\left(z^{\prime}\right)}{1-v^{2}}, \quad Q_{12}=Q_{21}=\frac{v E\left(z^{\prime}\right)}{1-v^{2}}, \quad Q_{66}=$ $\frac{E\left(z^{\prime}\right)}{2(1+v)}$.

The total in-plane force resultants and moments produced due to temperature rise are defined as:

$$
\begin{gathered}
N_{t}=\left\{\begin{array}{c}
N_{x} \\
N_{y} \\
N_{x y}
\end{array}\right\}=\int_{-h / 2-d}^{h / 2-d}\left\{\begin{array}{c}
\sigma_{x x} \\
\sigma_{y y} \\
\tau_{x y}
\end{array}\right\} d z^{\prime} \\
M_{t}=\left\{\begin{array}{c}
M_{x} \\
M_{y} \\
M_{x y}
\end{array}\right\}=\int_{-h / 2-d}^{h / 2-d}\left\{\begin{array}{c}
\sigma_{x x} \\
\sigma_{y y} \\
\tau_{x y}
\end{array}\right\} z^{\prime} d z^{\prime}
\end{gathered}
$$

The thermal thrust and moment can also be represented as:

$$
\begin{gathered}
\left\{N_{t x}\right\}=\left\{N_{t y}\right\}=\int_{-h / 2-d}^{h / 2-d}\{\beta\} \Delta T d z^{\prime}, \\
\left\{M_{t}\right\}=\int_{-h / 2-d}^{h / 2-d}\{\beta\} \Delta T z^{\prime} d z^{\prime} ; \\
\text { where }\{\beta\}=Q\{\alpha\}=\left\{\begin{array}{c}
\left(Q_{11}+Q_{12}\right) \alpha \\
\left(Q_{12}+Q_{22}\right) \alpha \\
0
\end{array}\right\} .
\end{gathered}
$$

\section{ENERGY EQUATIONS}

The potential strain energy $U^{e}$ of the plate element can be written in terms of the stress resultants and strain components through integrating by parts with respect to coordinates as follows

$$
U^{e}=U_{p s}^{e}+U_{t s}^{e}
$$

$U_{p s}^{e}$ Strain energy contribution from plane stress, $U_{t s}^{e}$ strain energy contribution from transverse shear stress $U^{e}=$ $\frac{1}{2} \iiint_{\nu} \varepsilon^{T} \sigma d v$.

$$
\begin{aligned}
U^{e}= & \frac{1}{2} \int_{a} \int_{-h / 2-d}^{h / 2-d}\left[\begin{array}{lll}
\varepsilon_{x} & \varepsilon_{y} & \varepsilon_{x y}
\end{array}\right] D\left\{\begin{array}{c}
\varepsilon_{x} \\
\varepsilon_{y} \\
\varepsilon_{x y}
\end{array}\right\} d z^{\prime} d a \\
& +\frac{k_{s h}}{2} \int_{a} \int_{-h / 2-d}^{h / 2-d}\left[\begin{array}{ll}
\gamma_{x} & \gamma_{y}
\end{array}\right] S\left\{\begin{array}{l}
\gamma_{x} \\
\gamma_{y}
\end{array}\right\} d z^{\prime} d a
\end{aligned}
$$

Here $k_{s h}$ is shear correction factor, $k_{s h}=\frac{5}{6-\left(v_{m} V_{m}+v_{c} V_{c}\right)}$, $v_{m}$, and $v_{c}$ represents the Poisson's ratio of metal and ceramic, $V_{m}$ and $V_{c}$ stands for the volume fraction of metal and ceramic, respectively. ${ }^{19}$

As the plate is exposed to thermal environment, it is subjected to in-plane stress resultants. Therefore, the work done by the in-plane forces produced due to temperature change produces out-of-plane displacement ' $w$ '.

Work done due to thermal loading of plate element is as follows:

$$
W_{t}^{e}=\frac{1}{2}\left(\int_{A} N_{t x}\left(\frac{\partial w}{\partial x}\right)^{2} d x+\int_{A} N_{t y}\left(\frac{\partial w}{\partial y}\right)^{2} d y\right)
$$

where $N_{t x}$ and $N_{t y}$ is the thermal thrust.

When the plate is rotating, it subjected to centrifugal forces. Therefore, the work is done by the plate centrifugal forces produced due to rotating speed. The centrifugal force on an element of the plate can be expressed as:

$$
F_{c}^{e}=\int_{x_{i}}^{x_{i}+l} \int_{-h / 2-d}^{h / 2-d} b \rho\left(z^{\prime}\right) \Omega^{2}(R+x) d z^{\prime} d x
$$

where $x_{i}$ is the distance of $i^{t h}$ node from axis of rotation, $\Omega$ $(\mathrm{rad} / \mathrm{s})$ is angular velocity of plate element and $R$ is the radius of the hub.

Work done by the centrifugal force is given by:

$$
W_{c}^{e}=\frac{1}{2} F_{c}^{e} w^{2} d x=\frac{1}{2}\{q\}^{T}\left[k_{c}\right]\{q\} .
$$

The kinetic energy of the plate element can be expressed as follows

$$
T^{e}=\frac{1}{2} \iint \rho h \dot{w}^{2} d A
$$




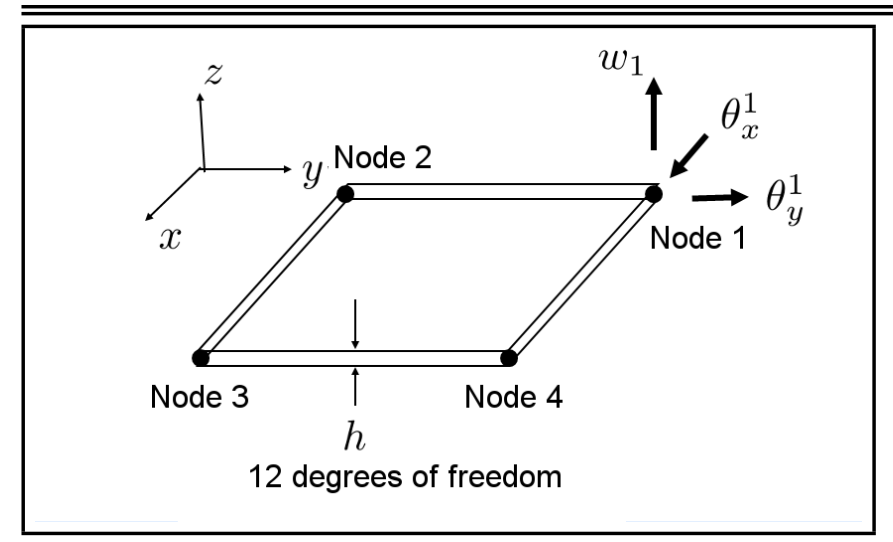

Figure 5. The finite element model of the rectangular element.

\subsection{Finite Element Model Formulation}

In the present FEM formulation, the derivatives are assumed as independent field variables by considering $w, \theta_{x}$, and $\theta_{y}$ as separate DOFs. A four-noded rectangular element is employed for finite-element modelling as shown in Fig. 5. Over each of the elements, the displacement vector is expressed by:

$$
\begin{aligned}
w & =\sum_{i=1}^{n} N_{i} w_{i} \\
\theta_{x} & =\sum_{i=1}^{n} N_{i} \theta_{x}^{i} ; \\
\theta_{y} & =\sum_{i=1}^{n} N_{i} \theta_{y}^{i}
\end{aligned}
$$

where $N_{i}$ are the appropriate shape functions, $n$ represents number of nodes in the element.

\subsection{Strain energy of the plate element}

The strain energy of the FGM plate element is given by

$$
U^{e}=\frac{1}{2}\left\{q^{e}\right\}^{T}\left[K_{s}^{e}\right]\left\{q^{e}\right\}
$$

where $\left[K_{s}^{e}\right]$ and $\left\{q^{e}\right\}$ are defined as element stiffness matrix and displacement vector of the element, respectively. The element stiffness matrix is derived as:

$$
K_{s}^{e}=\int_{v}\left[B_{b}\right]^{T}\left[D_{b}\right]\left[B_{b}\right] d v+\int_{v}\left[B_{s}\right]^{T}\left[D_{s}\right]\left[B_{s}\right] d v
$$

where $\left[B_{b}\right]=\left[\begin{array}{ccc}0 & \frac{\partial}{\partial x} & 0 \\ 0 & 0 & \frac{\partial}{\partial y} \\ 0 & \frac{\partial}{\partial y} & \frac{\partial}{\partial x}\end{array}\right]\left[N_{i}\right], \quad\left[B_{s}\right]=$ $\left[\begin{array}{ccc}\frac{\partial N_{i}}{\partial x} & N_{i} & 0 \\ \frac{\partial N_{i}}{\partial y} & 0 & N_{i}\end{array}\right]$

$$
W_{T}^{e}=\int q^{(e) T} \lambda_{T}\left[K_{t}^{e}\right]^{e} q^{(e)} d a ;
$$

where $\lambda_{T}$ and $\left[K_{t}^{e}\right]$ are defined as thermal buckling load parameters and the element geometric stiffness matrix for the element, respectively. The element geometric stiffness matrix is derived as:

$$
\left[K_{t}^{e}\right]=N_{t x} \iint\left(\frac{\partial^{2} w}{\partial x^{2}}\right) d x+N_{t y} \iint\left(\frac{\partial^{2} w}{\partial y^{2}}\right) d y .
$$

Here, $N_{t x}$ and $N_{t y}$ are thermal thrust along x and y axis. The Element centrifugal stiffness matrix is defined as:

$$
\begin{aligned}
W_{c}^{e}=\frac{1}{2}\{q\}^{(e)^{T}}\left[K_{c}^{e}\right]\{q\}^{(e)} & \\
{\left[K_{c}^{e}\right] } & =F_{c} \iint[N]^{T}[N] d x d y .
\end{aligned}
$$

\subsection{Kinetic energy of the plate}

The kinetic energy of the vibrating plate may be written as

$$
\begin{aligned}
T^{e}=\frac{1}{2} \int_{v}\left\{\dot{q}^{e}\right\}^{T}\left[M^{e}\right]\left\{\dot{q}^{e}\right\} ; \\
\delta=L / W, \varsigma=R / L, \lambda=\Omega T, \eta=h / L ; \\
\varpi=\omega L^{2} \sqrt{\rho h / D} \\
\{\beta\}=Q\{\alpha\}=\left\{\begin{array}{l}
\left(Q_{11}+Q_{12}\right) \alpha \\
\left(Q_{12}+Q_{22}\right) \alpha \\
0
\end{array}\right\} .
\end{aligned}
$$

Here, $\left[M^{e}\right]$ is the mass matrix of the element.

The Element mass matrix is derived as $\left[M^{e}\right]=\int N^{T} \rho N d v$ in which $N$ is the shape function matrix given as: ${ }^{v} N_{(3 X 12)}=$ $\left[\begin{array}{cccccccccccc}N_{1} & 0 & 0 & N_{2} & 0 & 0 & N_{3} & 0 & 0 & N_{4} & 0 & 0 \\ 0 & N_{1} & 0 & 0 & N_{2} & 0 & 0 & N_{3} & 0 & 0 & N_{4} & 0 \\ 0 & 0 & N_{1} & 0 & 0 & N_{2} & 0 & 0 & N_{3} & 0 & 0 & N_{4}\end{array}\right]$
$N_{1}=\frac{(1-\xi)(1-\eta)}{4}, N_{2}=\frac{(1+\xi)(1-\eta)}{4}, N_{3}=\frac{(1+\xi)(1+\eta)}{4}, N_{4}=$ $\frac{(1-\xi)(1+\eta)}{4}$, where $\xi=x / a, \eta=y / b, a$ and $b$ are the element length and width.

\section{GOVERNING EQUATIONS OF MOTION}

The element equation of motion for a rotating FGM plate in thermal environment is obtained by using Hamilton's principle:

$$
\delta \int_{t_{1}}^{t_{2}}\left(U^{e}-T^{e}\right) d t=0
$$

By dividing the plate into a number of elements, the potential energy and the kinetic energy for an element can be written in terms of element nodal displacement vector as:

$$
\begin{gathered}
U^{e}=\frac{1}{2}\left\{q^{e}\right\}^{T}\left(\left[K_{s}^{e}\right]+\left[K_{c}^{e}\right]\right)\left\{q^{e}\right\}-\frac{1}{2}\left\{q^{e}\right\}^{T}\left[K_{t}^{e}\right]\left\{q^{e}\right\}= \\
\frac{1}{2}\left\{q^{e}\right\}^{T}\left[K_{e}^{e f}\right]\left\{q^{e}\right\} ; \\
T^{e}=\frac{1}{2}\left\{\dot{q}^{e}\right\}^{T}\left[M^{e}\right]\left\{\dot{q}^{e}\right\} .
\end{gathered}
$$

Substituting Eq. (31) and Eq. (32) in Eq. (30) and carrying out the variation the equation of motion for the element can be written as

$$
\left[M^{e}\right]\left\{\ddot{q}^{e}\right\}+\left[K_{e}^{e f}\right]\left\{q^{e}\right\}=0 ;
$$

where $\left[K_{e}^{e f}\right]=\left[K_{s}^{e}\right]+\left[K_{c}^{e}\right]-\left[K_{t}^{e}\right]$ is the effective element stiffness matrix. Assembling the element matrices the equation of motion in terms of global matrices and global displacement vector can be written as follows:

$$
[M]\{\ddot{q}\}+\left[K^{e f}\right]\{q\}=0 ;
$$


Table 1. Compression of the first five natural frequencies parameter by the present and Yoo and Kim. ${ }^{5}$

\begin{tabular}{|c|c|c|c|c||}
\hline $\begin{array}{c}\text { Dimensionless } \\
\text { angular speed }\end{array}$ & Mode & $\begin{array}{c}\text { Yoo } \\
\text { and Kim }\end{array}$ & $\begin{array}{c}\text { Yoo and Kim } \\
\text { Southwell method }\end{array}$ & $\begin{array}{c}\text { Present } \\
\text { method }\end{array}$ \\
\hline$\lambda=1$ & 1 & 3.65280 & 3.65427 & 3.5567 \\
\hline & 2 & 8.64590 & 8.64350 & 8.6205 \\
\hline & 3 & 21.53372 & 21.55690 & 21.4360 \\
\hline & 4 & 27.38478 & 27.47469 & 27.2818 \\
\hline & 5 & 31.21853 & 31.38484 & 31.0489 \\
\hline$\lambda=2$ & 1 & 4.11312 & 4.112807 & 4.1190 \\
\hline & 2 & 9.00317 & 8.98438 & 9.4010 \\
\hline & 3 & 21.96654 & 22.01202 & 22.1913 \\
\hline & 4 & 27.62312 & 27.86609 & 28.1300 \\
\hline & 5 & 31.58544 & 32.17962 & 31.6746 \\
\hline
\end{tabular}

Table 2. Compression of the first five natural frequencies parameter by the present and Yoo and Kim, ${ }^{5} \delta=1, \varsigma=1$.

\begin{tabular}{||c|c|c|c|c||}
\hline $\begin{array}{c}\text { Dimensionless } \\
\text { angular speed }\end{array}$ & Mode & $\begin{array}{c}\text { Yoo } \\
\text { and Kim }^{5}\end{array}$ & $\begin{array}{c}\text { Yoo and Kim } \\
\text { Southwell method }\end{array}$ & $\begin{array}{c}\text { Present } \\
\text { method }\end{array}$ \\
\hline$\lambda=1$ & 1 & 3.86185 & 3.80871 & 3.9703 \\
\hline & 2 & 8.73585 & 8.72801 & 9.1854 \\
\hline & 3 & 21.71968 & 21.74132 & 21.9558 \\
\hline & 4 & 27.42572 & 27.62624 & 27.9096 \\
\hline & 5 & 31.36240 & 31.77682 & 31.4956 \\
\hline$\lambda=2$ & 1 & 4.81385 & 4.63956 & 5.1079 \\
\hline & 2 & 9.34355 & 9.30544 & 10.9537 \\
\hline & 3 & 22.79012 & 22.72604 & 23.7636 \\
\hline & 4 & 27.79012 & 28.45908 & 29.9878 \\
\hline & 5 & 32.14933 & 33.68323 & 33.0389 \\
\hline
\end{tabular}

where $\left[K^{e f}\right]$ is effective global effective stiffness matrix, and $[M]$ is the global mass matrix. Eigen values of Eq. (34) gives the natural frequencies of the plate.

\section{RESULTS AND DISCUSSION}

The numerical results of thermo-mechanical vibration analysis of the rotating FGM plates are calculated using the proposed mathematical model using FEM. A four node rectangular element, with 12 DOFs per element has been used to discretize the plate. A computer program has been developed in an environment created using MATLAB. The validation and efficiency of the proposed algorithm is examined by comparing the results with those available in the literature.

\subsection{Validations of the Results}

\subsubsection{Rotating Plate Frequency}

The rotating isotropic material plate frequency parameters obtained by using the present method are compared with those obtained by Yoo and $\mathrm{Kim}^{5}$ to show the reliability of method of calculation presented in this paper. The rotating plate frequency parameters obtained are shown in Tables 1 and 2. As shown in these tables, the results obtained by using the present method are in reasonable agreement with those obtained by Southwell's method. ${ }^{5}$

Here, in all the presented tables and figures, $\delta, \varsigma, \lambda, \eta$, and $\varpi$ denote the aspect ratio, hub radius ratio, dimensionless rotation speed thickness ratio, and natural frequency parameter respectively. They are defined as: $\delta=L / W, \varsigma=R / L, \lambda=\Omega T$, $\eta=h / L, \varpi=\omega L^{2} \sqrt{\rho h / D}$ where $D=E h^{3} / 12\left(1-\nu^{2}\right)$.

\subsubsection{Comparison of Natural Frequencies of FGM Plates}

Table 3 shows the SUS304, $\mathrm{Si}_{3} \mathrm{~N}_{4}$, and $\mathrm{Al}_{2} \mathrm{O}_{3}$ temperaturedependent properties of the FGM constituents that have been used for the computation of the present analysis.

Table 4 shows the comparison of the first five natural frequency parameter of FGM plate obtained by applying first order shear deformation theory in this study and those obtained by Huang and Shen using the higher-order shear deformation plate theory. ${ }^{14}$ The results show good agreement. The difference is due to the higher order displacement functions that are used in the reference results.

Table 4 shows the effect of volume fraction index $\mathrm{k}$ on the natural frequency parameter of FGM $\left(\mathrm{Si}_{3} \mathrm{~N}_{4} / \mathrm{SUS} 304\right)$ plates under thermal loading condition, $T_{m}=300 \mathrm{~K}, T_{c}=400 \mathrm{~K}$, and $\nu=0.28$. Temperature-dependent and temperatureindependent material properties are considered for this analysis. It can be seen that the natural frequency parameter of the FGM plate decreases with the increase of volume fraction index $k$.

\subsection{The Rotating Ordinary FGM Plate Natural Frequencies}

Now we study the FGM $\left(\mathrm{Al}_{2} \mathrm{O}_{3} / \mathrm{SUS304}\right)$ plate for this analysis. For this numerical study, the following material properties are consider as:

SUS304, $\rho=8.166 \mathrm{~kg} / \mathrm{m}^{3}, E=207.78 \times 10^{9} \mathrm{~N} / \mathrm{m}^{2}, \vartheta=0.3$ $\mathrm{Al}_{2} \mathrm{O}_{3}, \rho=3800 \mathrm{~kg} / \mathrm{m}^{3}, E=320.024 \times 10^{9} \mathrm{~N} / \mathrm{m}^{2}, \vartheta=0.3$.

Figures 6(a) and 6(b) show the variations of the first five natural frequencies of rotating FGM plates with an aspect ratio of 1 , a thickness ratio of 0.01 , and the a hub radius ratio of 0 and 1. According to these figures, it can be seen that the first five natural frequencies decrease as the volume fraction index increases at rotating speed 100 RPM. Figures 7 shows the first five natural frequencies variation with hub radius ratio. The increasing hub radius increases the first five natural frequencies. The variation of natural frequency versus rotating speed for FGM plates is plotted in Figs. 8(a) to 8(d). These figures are plotted for $1^{\text {st }}, 2^{\text {nd }}, 3^{\text {rd }}$, and $4^{\text {th }}$ mode frequencies of FGM plate, for different power law index values $(k=0,0.5,2)$ at rotating speed 100 RPM. As expected, the natural frequencies increase as the angular speed increases. This results from the centrifugal inertia force which increases as the hub radius and the angular speed increase.

\subsection{Natural Frequencies of the Rotating Temperature Dependent FGM Plate}

Here, the upper surface of the temperature dependent FGM plate is maintained at a maximum of $800 \mathrm{~K}$, which is ceramicrich, whereas the lower one is metal-rich at $300 \mathrm{~K}$. The aspect ratio $(L / W)$ is taken as 1 . The temperature eld is assumed to vary in the thickness direction only and is determined by the steady-state heat conduction equation across the thickness of the plate.

Figures 9(a) to 9(d) show the variation of first four frequencies of the FGM plate in a thermal environment with a temper- 
I. Ramu, et al.: FREE VIBRATION ANALYSIS OF ROTATING PLATES IN HIGH THERMAL ENVIRONMENTS USING THE FINITE ELEMENT...

Table 3. Temperature-dependent coefficients of the elastic modulus $E$ [GPa] and thermal expansion coefficient $[1 / \mathrm{K}]$ for ceramics and metals. ${ }^{14}$

\begin{tabular}{|c|c|c|c|c|c|c|}
\hline \hline Materials & Proprieties & $P_{0}$ & $P_{-1}$ & $P_{1}$ & $P_{2}$ & $P_{3}$ \\
\hline \multirow{2}{*}{ SUS304 } & $E_{m}$ & $201.04 \times 10^{9}$ & 0 & $3.079 \times 10^{-4}$ & $-6.534 \times 10^{-7}$ & 0.0 \\
\cline { 2 - 7 } & $\alpha$ & $12.33 \times 10^{-6}$ & 0 & $8.086 \times 10^{-4}$ & 0.0 & 0.0 \\
\hline \multirow{2}{*}{$\mathrm{Si}_{3} \mathrm{~N}_{4}$} & $E_{c}$ & $348.43 \times 10^{9}$ & 0 & $-3.079 \times 10^{-4}$ & $2.160 \times 10^{-7}$ & $-8.946 \times 10^{-11}$ \\
\cline { 2 - 7 } & $\alpha$ & $5.8723 \times 10^{-6}$ & 0 & $9.095 \times 10^{-4}$ & 0.0 & 0.0 \\
\hline \multirow{2}{*}{$\mathrm{Al}_{2} \mathrm{O}_{3}$} & $E_{c}$ & $349.55 \times 10^{9}$ & 0 & $-3.853 \times 10^{-4}$ & $4.027 \times 10^{-7}$ & $-1.673 \times 10^{-11}$ \\
\cline { 2 - 7 } & $\alpha$ & $6.826 \times 10^{-6}$ & 0 & $1.838 \times 10^{-4}$ & 0.0 & 0.0 \\
\hline
\end{tabular}

Table 4. Comparison of the first five natural frequency parameter $\Omega=\omega\left(L^{2} / h\right)\left[\rho_{m}\left(1-v^{2}\right) / E_{m}\right]^{1 / 2}$ for FGM (Si $\left.\mathrm{N}_{4} / \mathrm{SUS}_{304}\right)$ plates in thermal environments.

\begin{tabular}{|c|c|c|c|c|c|c|c|}
\hline & \multirow{2}{*}{$\begin{array}{l}\text { Index } \\
\text { value }\end{array}$} & \multicolumn{6}{|c|}{ First five natural frequencies parameter } \\
\hline & & & 1 & 2 & 3 & 4 & 5 \\
\hline \multirow{8}{*}{$\begin{array}{l}\text { Temperature } \\
\text { independent }\end{array}$} & \multirow{2}{*}{$\mathrm{Si}_{3} \mathrm{~N}_{4}$} & Huang and Shen ${ }^{14}$ & 12.382 & 29.243 & 29.243 & 44.072 & 53.105 \\
\hline & & Present & 12.633 & 29.717 & 29.717 & 44.801 & 54.668 \\
\hline & \multirow{2}{*}{0.5} & Huang and Shen ${ }^{14}$ & 8.641 & 20.316 & 20.316 & 30.682 & 37.007 \\
\hline & & Present & 8.798 & 20.698 & 20.698 & 31.205 & 38.078 \\
\hline & \multirow{2}{*}{1} & Huang and Shen ${ }^{14}$ & 7.514 & 17.694 & 17.694 & 26.717 & 32.242 \\
\hline & & Present & 7.676 & 18.060 & 18.060 & 27.227 & 33.225 \\
\hline & \multirow{2}{*}{2} & Huang and Shen ${ }^{14}$ & 6.728 & 15.836 & 15.836 & 23.893 & 28.816 \\
\hline & & Present & 6.795 & 15.987 & 15.987 & 24.103 & 29.412 \\
\hline \multirow{8}{*}{$\begin{array}{c}\text { Temperature } \\
\text { dependent }\end{array}$} & \multirow{2}{*}{$\mathrm{Si}_{3} \mathrm{~N}_{4}$} & Huang and Shen ${ }^{14}$ & 12.397 & 29.083 & 29.083 & 43.835 & 52.822 \\
\hline & & Present & 12.319 & 29.316 & 29.316 & 44.320 & 54.138 \\
\hline & \multirow{2}{*}{0.5} & Huang and Shen ${ }^{14}$ & 8.615 & 20.215 & 20.215 & 30.530 & 36.824 \\
\hline & & Present & 8.522 & 20.370 & 20.370 & 30.827 & 37.670 \\
\hline & \multirow{2}{*}{1} & Huang and Shen ${ }^{14}$ & 7.474 & 17.607 & 17.607 & 26.590 & 32.088 \\
\hline & & Present & 7.412 & 17.755 & 17.755 & 26.883 & 32.857 \\
\hline & \multirow{2}{*}{2} & Huang and Shen ${ }^{14}$ & 6.693 & 15.762 & 15.762 & 23.786 & 28.686 \\
\hline & & Present & 6.540 & 15.702 & 15.702 & 23.788 & 29.080 \\
\hline
\end{tabular}

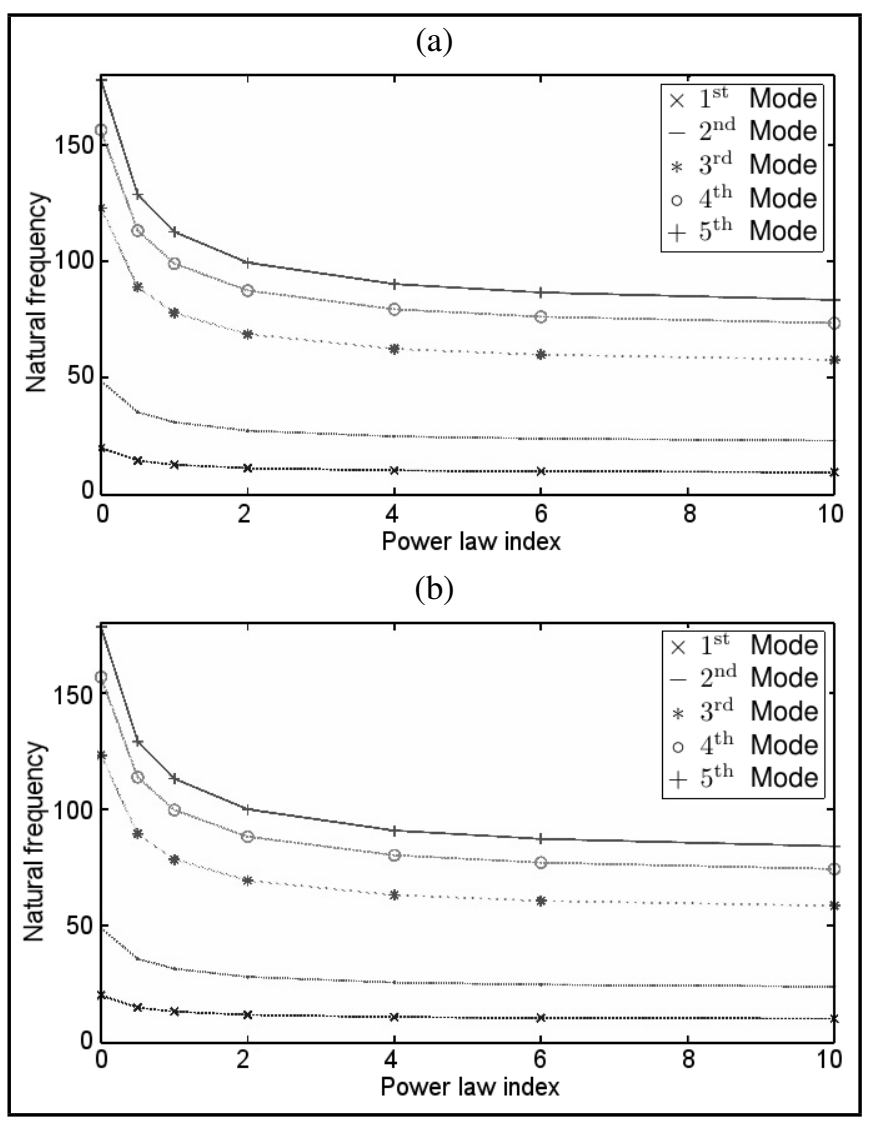

Figure 6. (a) The rotating ordinary FGM plate for the first five natural frequencies variation with a power law index value at speed $100 \mathrm{RPM}$ and $R / L=0$. (b) The rotating ordinary FGM plate for the first five frequencies parameter variation with a power law index value at speed $100 \mathrm{RPM}$ and $R / L=1$

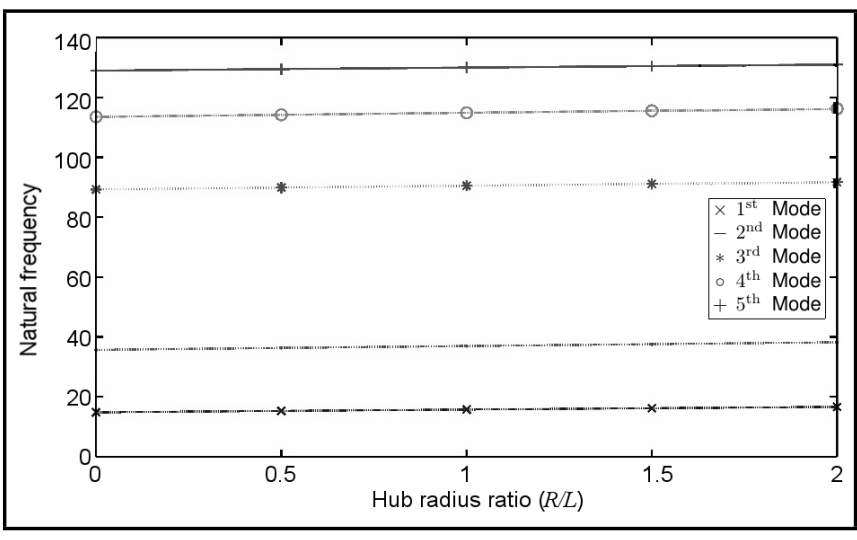

Figure 7. Rotating ordinary FGM plate for the first five natural frequencies variation with the hub radius ratio $(R / L)$ at speed $150 \mathrm{RPM}$ and $k=0.5$.

ature change $(0-500 \mathrm{~K})$; the frequencies reduce as the temperature change increases. It is shown that the effect of temterature change on the values of first four frequencies decrease with an increase of the temperature difference from 0 to $500 \mathrm{~K}$. This tendency is observed because Young's modulus decreases as temperature goes up and consequently, the lesser Young's modulus lowers the natural frequencies.

Figures 10(a) to 10(d) show the variation of first four mode frequencies of FGM plate in thermal environment with hub radius ratio, for different power law indices $k=0,0.5$ and 2 at rotating speed 150 RPM. From Figs. 10(a) to 10(d), one can observe that the first four frequencies increase with increase in hub radius ratio. This happens due to centrifugal stiffening with an increased hub radius.

Figures 11(a) to 11(d) depict the first four frequencies versus the rotating speed for different power law index values $(k=0$, $0.5,2)$, respectivly. The increasing rotating speed increases the 


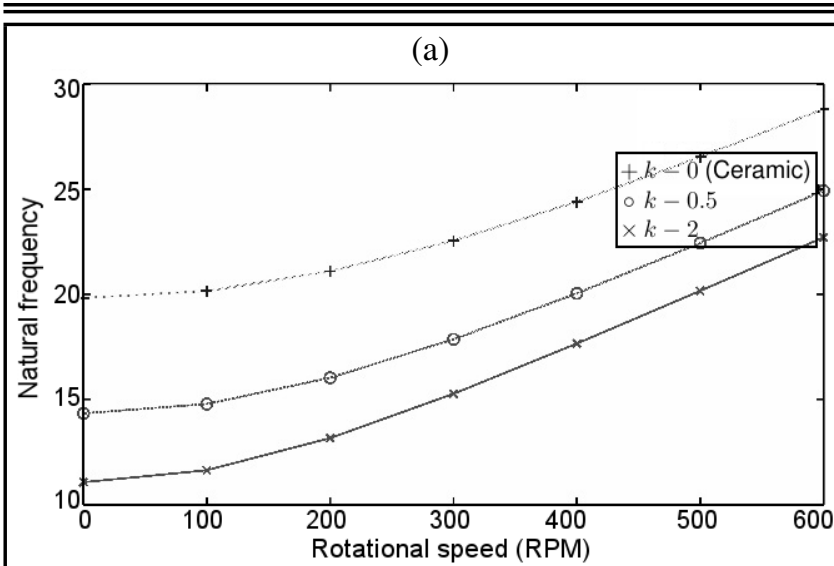

(b)

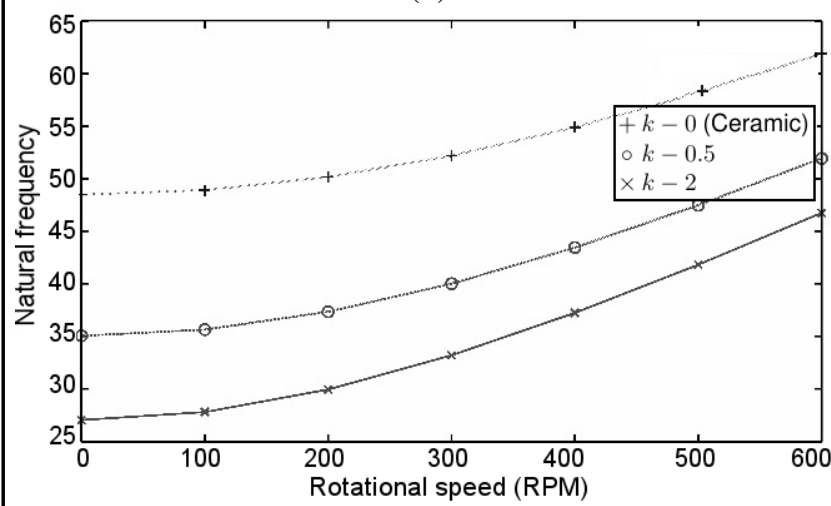

(c)

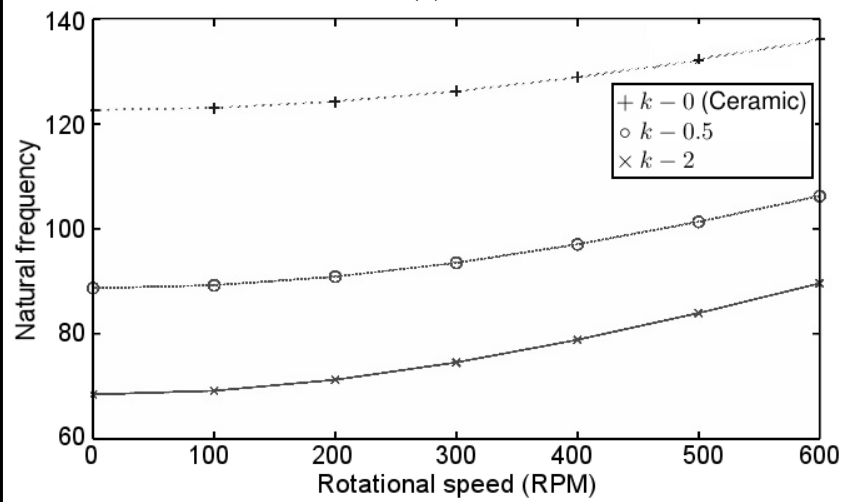

(d)

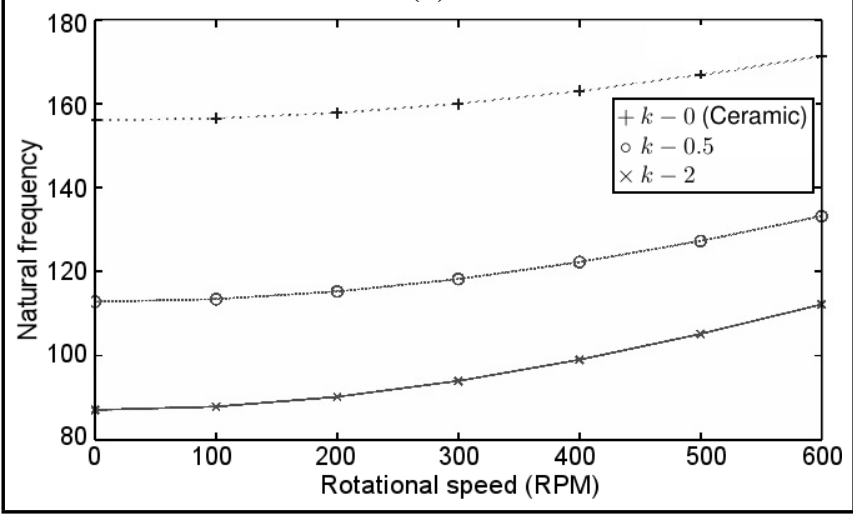

Figure 8. (a) Variation of first mode frequency with the rotational speed at the hub radius $(R / L=0.5)$. (b) Variation of second mode frequency with the rotational speed at the hub radius $(R / L=0.5)$. (c) Variation of third mode frequency with the rotational speed at the hub radius $(R / L=0.5)$. (d) Variation of fourth mode frequency with the rotational speed at the hub radius $(R / L=0.5)$.

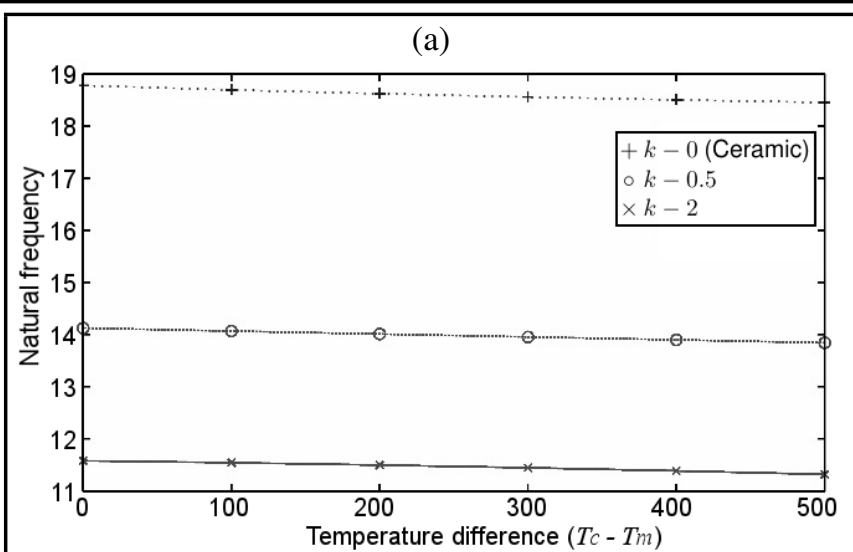

(b)

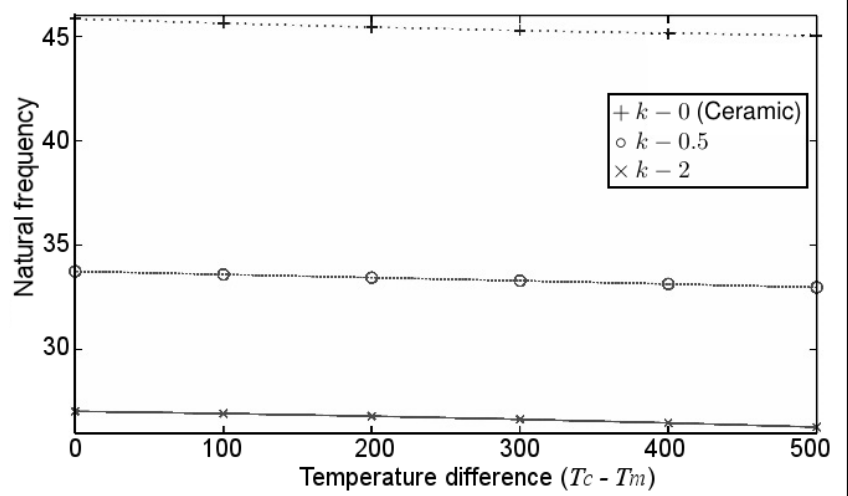

(c)

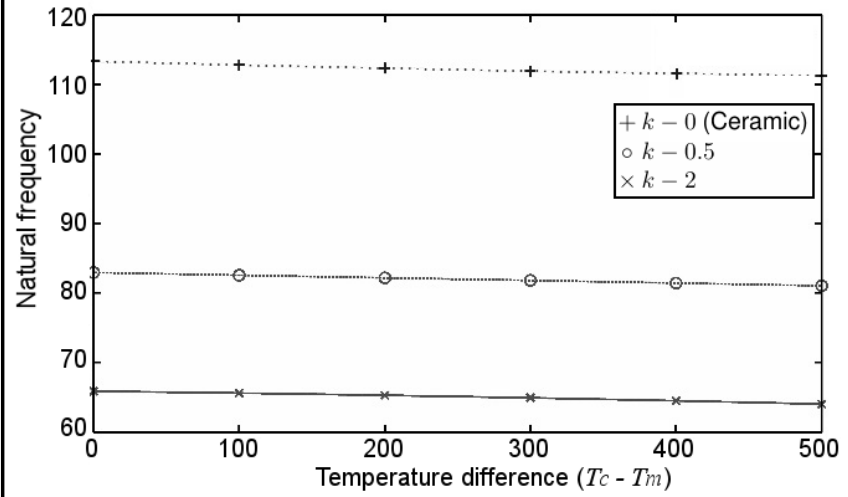

(d)

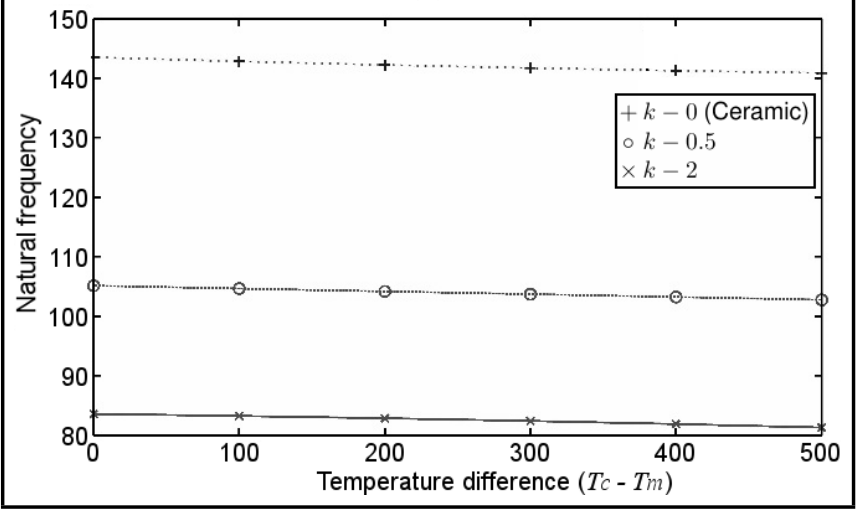

Figure 9. (a) Variation of the first natural frequency of the rotating temperature dependent FGM plate with a temperature change (speed 150 RPM and $R / L=$ 0.4 ). (b) Variation of the second natural frequency of the rotating temperature dependent FGM plate with a temperature change (speed $150 \mathrm{RPM}$ and $R / L=$ 0.4 ). (c) Variation of the third natural frequency of the rotating temperature dependent FGM plate with a temperature change (speed 150 RPM and $R / L=$ $0.4)$. (d) Variation of the fourth natural frequency of the rotating temperature dependent FGM plate with a temperature change (speed $150 \mathrm{RPM}$ and $R / L=$ $0.4)$. 


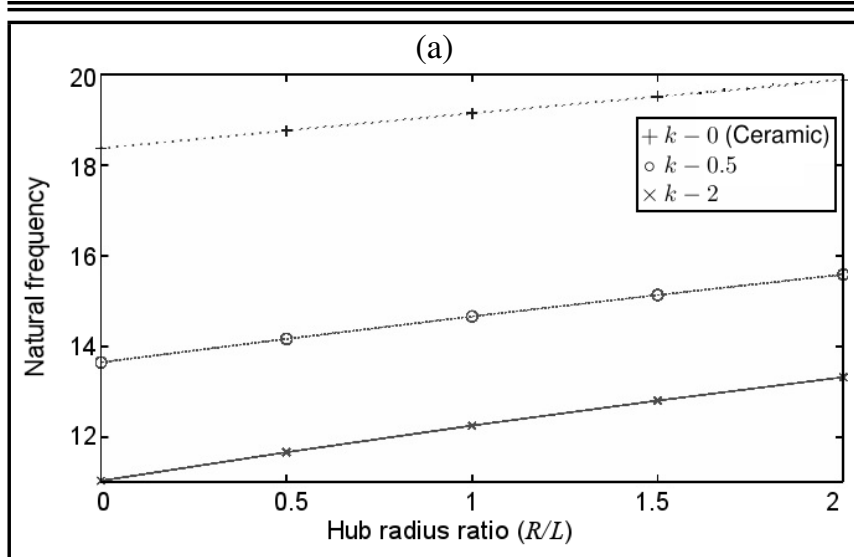

(b)

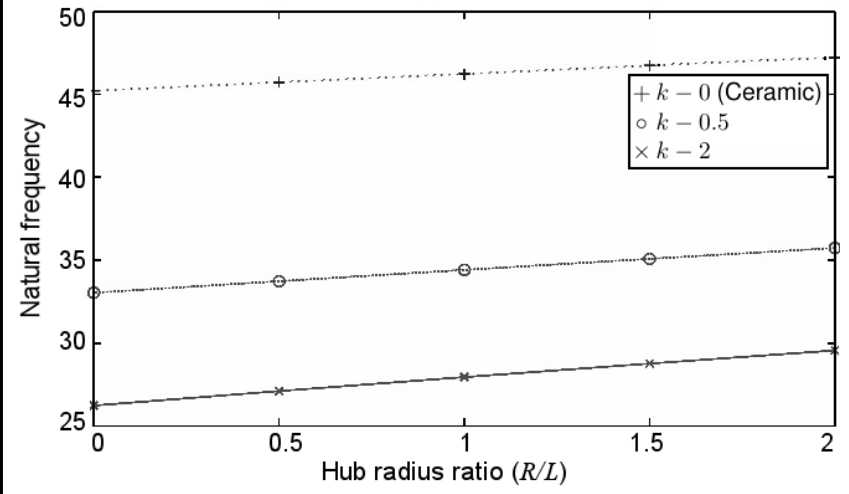

(c)

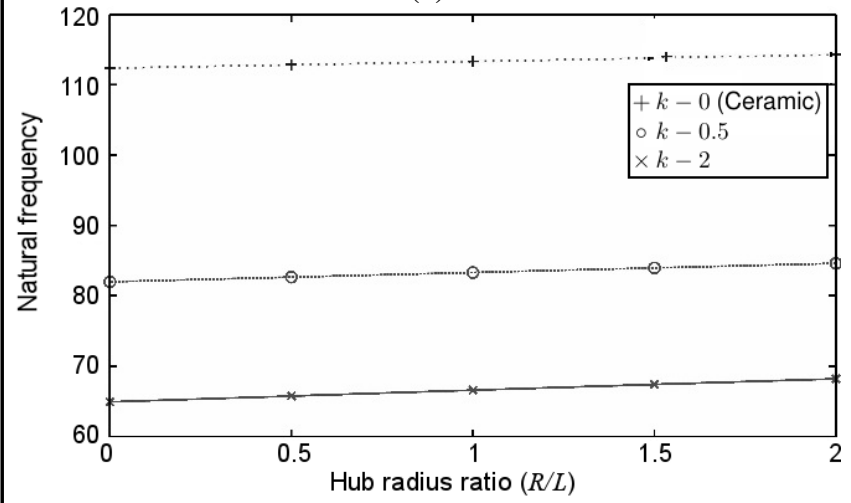

(d)

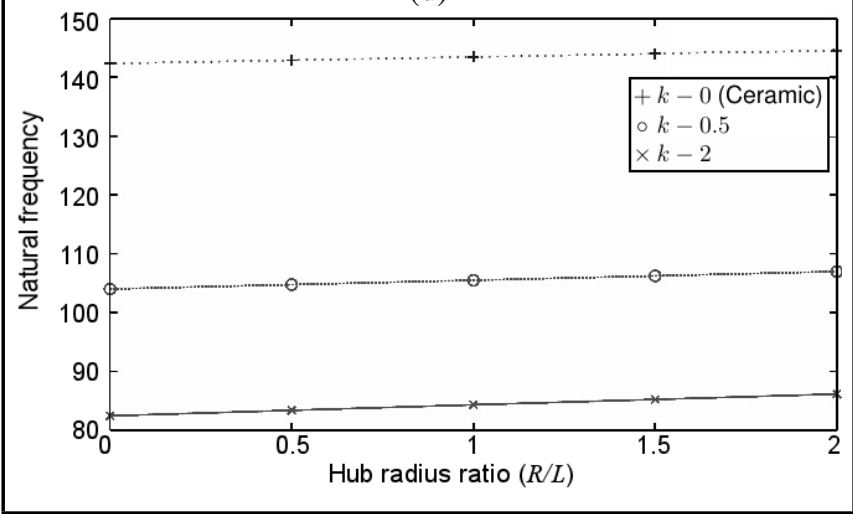

Figure 10. (a )Variation of the rotating temperature dependent FGM plate first natural frequency with a hub radius at speed 150 RPM and temperature difference $100 \mathrm{~K}$. (b) Variation of the rotating temperature dependent FGM plate second natural frequency with a hub radius at speed 150 RPM and temperature difference $100 \mathrm{~K}$. (c) Variation of the rotating temperature dependent FGM plate third natural frequency with a hub radius at speed 150 RPM and temperature difference $100 \mathrm{~K}$. (d) Variation of the rotating temperature dependent FGM plate fourth natural frequency with a hub radius at speed 150 RPM and temperature difference $100 \mathrm{~K}$. (a)

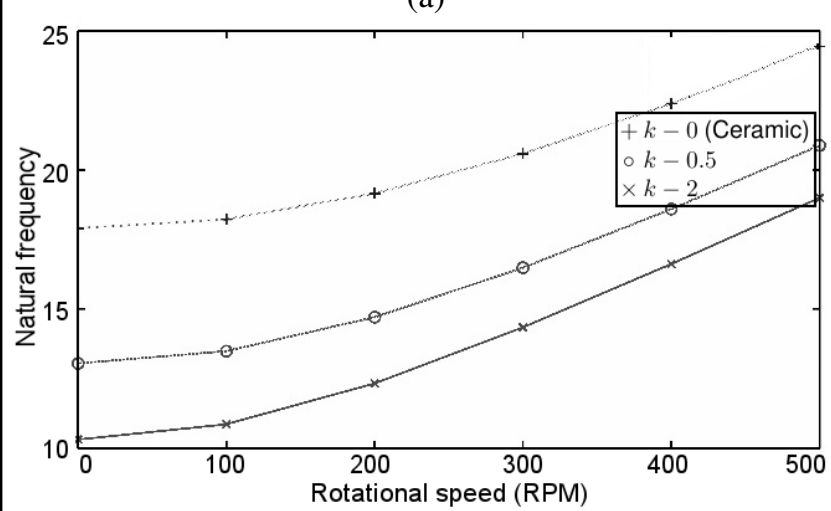

(b)

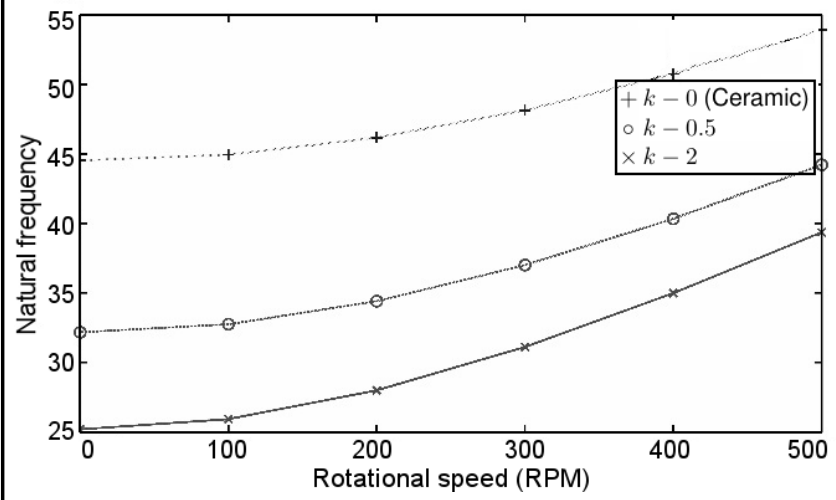

(c)

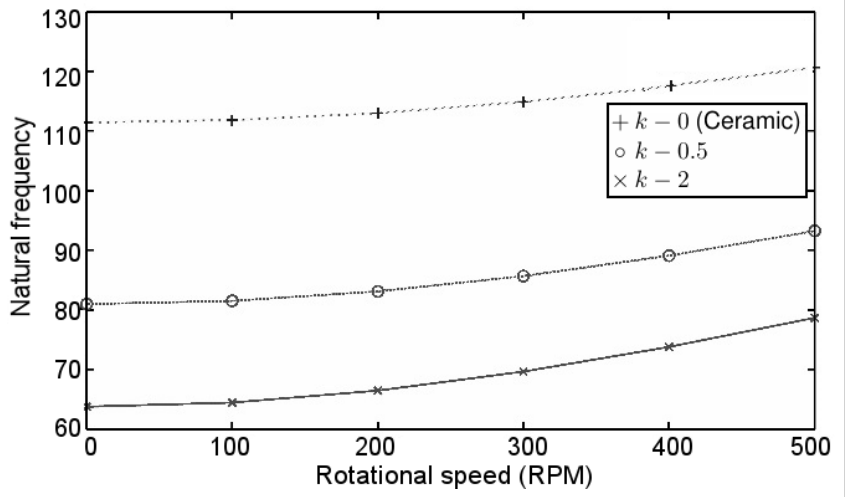

(d)

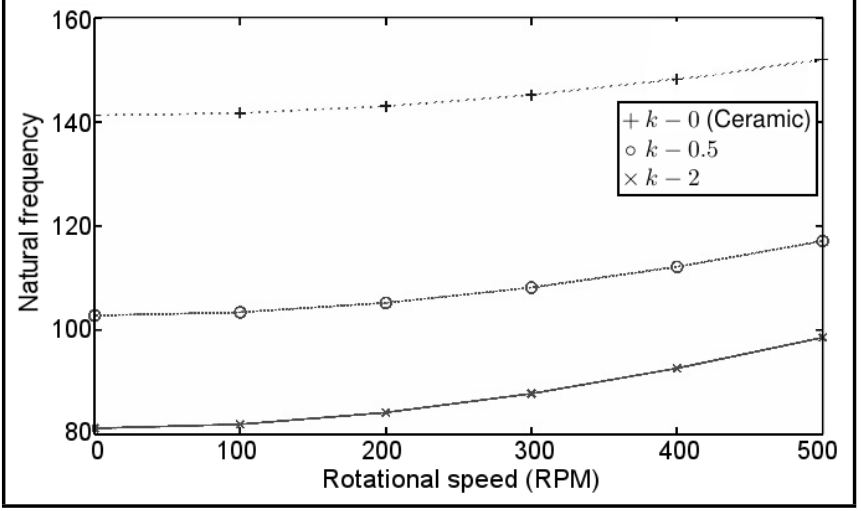

Figure 11. (a) Variation of the first natural frequency of rotating temperature dependent FGM plate with a rotating speed (temperature change $200 \mathrm{~K}$ and $R / L=0.4$ ). (b) Variation of the second natural frequency of rotating temperature dependent FGM plate with a rotating speed (temperature change $200 \mathrm{~K}$ and $R / L=0.4$ ). (c) Variation of the third natural frequency of rotating temperature dependent FGM plate with a rotating speed (temperature change $200 \mathrm{~K}$ and $R / L=0.4$ ). (d) Variation of the first fourth natural frequency of rotating temperature dependent FGM plate with a rotating speed (temperature change $200 \mathrm{~K}$ and $R / L=0.4$ ). 
inertia force, it causes increase in natural frequencies. Thus, from these Figs. 11(a) to 11(d) it is concluded that increase in rotational speed increases the natural frequencies irrespective of the power law index value.

\section{CONCLUSIONS}

Flapwise bending vibration characteristics of rotating functionally graded plates have been presented. The analysis was carried out using the FSDT along with FEM. The validation studies have been carried out to establish the correctness of the present formulation and calculation. Four parameters are identified through the proposed numerical experiments: power law index, the hub radius, the angular speed, and temperature rise. The effect of these parameters on the natural frequency of rotating FGM plates are studied in detail. Observations from the analysis: the rotating FGM plate natural frequencies increase with the increase in the angular rotating speed and hub radius. The natural frequencies reduces with the increase in power law index value and temperature rise.

\section{REFERENCES}

1 Ramamurti, V. and Kielb, R. Natural frequencies of twisted rotating plates, Journal of Sound and Vibration, 97 (3), 429-449, (1984).

2 Sreenivasa Murthy, S. and Ramamurti, V. Coriolies effect on the vibration of flat rotating low aspect ratio cantilever plates, Journal of Strain Analysis for Engineering Design, 16, 97-106, (1981).

3 Wang, J. T. S., Shaw, D., and Mahrenholtz, O. Vibration of rotating rectangular plates, Journal of Sound and Vibration, 112 (3), 455-468,(1987).

4 Yoo, H. H., Park, J. H., and Park, J. Vibration analysis of rotating pre-twisted blades, Computers and Structures, 79, 1811-1819, (2001).

5 Yoo, H. H. and Kim, S. K. Flapwise bending vibration of rotating plates, International Journal for Numerical Methods in Engineering, 55, 785-802, (2002).

6 Yoo, H. H. and Pierre, C. Modal characteristic of a rotating rectangular cantilever plate, Journal of Sound and Vibration, 259 (1), 81-96, (2003).

7 Hong Seok Lim and Hong Hee Yoo, Modal analysis of cantilever plates undergoing accelerated in-plane motion, Journal of Sound and Vibration, 297, 880-894, (2006).

8 Hashemi, S. H., Farhadi, S., and Carra, S. Free vibration analysis of rotating thick plates, Journal of Sound and Vibration, 323, 366-384, (2009).
9 Zhang, D. and Zhou, Y. A theoretical analysis of FGM thin plates based on physical neutral surface, Computational Materials Science, 44, 716-720, (2008).

10 Chi, S. and Chung, Y. Mechanical behaviour of functionally graded material plates under transverse loadPart II: Numerical results, International Journal of Solids and Structures, 43, 3675-3691, (2006).

11 Croce, L and Venini, P. Finite elements for functionally graded ReissnerMindlin plates, Computer Methods Applied Mechanical Engineering, 193, 705-725, (2004).

12 Mohammad Talha, and Singh, B. N. Static response and free vibration analysis of FGM plates using higher order shear deformation theory, Applied Mathematical Modelling, 34, 3991-4011, (2010).

13 Hosseini-Hashemi, Sh. Fadaee, M., and Atashipour, S. R. Study on the free vibration of thick functionally graded rectangular plates according to a new exact closed-form procedure, Composite Structures, 93, 722-735, (2011).

14 Huang, $X$ and Shen, H. Nonlinear vibration and dynamic response of functionally graded plates in thermal environments, International Journal of Solids and Structures, 41, 2403-2427, (2004).

15 Yang, J. and Shen, H. S. Vibration characteristics and transient response of shear-deformable functionally graded plates in thermal environments, Journal of Sound and vibration, 255 (3), 579-602, (2002).

16 Talha, M. and Singh, B. N. Thermo-mechanical induced vibration characteristics of shear deformable functionally graded ceramicmetal plates using the finite element method, Proceedings of the Institution of Mechanical Engineers, Part C: Journal of Mechanical Engineering Science, 225, 50-67, (2011).

17 Kim, Y. Temperature dependent vibration analysis of functionally graded rectangular plates, Journal of Sound and Vibration, 284, 531-549, (2005).

18 Ramu, Inala and Mohanty, S. C. Dynamic stability of functionally graded material plates in high temperature environment, International Journal of Aerospace and Lightweight Structures, Accepted (2014).

19 Efraim, E. Eisenberger, M. Exact vibration analysis of variable thickness thick annular isotropic and FGM plates, Journal of Sound and Vibration, 299, 720-738, (2007).

20 Praveen, G. N. and Reddy, J. N. Nonlinear transient thermo elastic analysis of functionally graded ceramicmetal PLATES, International Journal of Solids and Structures, 35, 4457-4476, (1998). 